\title{
100 hours of astronomy at the Mexican northern border
}

\section{To the Editor - The International}

Astronomical Union (IAU) encourages inclusivity ${ }^{1}$ and information technology presents tools that can make astronomy accessible to all. By using social media, our outreach proposal intends to inspire the next generation of astronomers. By using apps and social media combined with traditional talks, interaction between the public and researchers can be facilitated, and a more diverse audience can be reached, in line with the IAU's mission to build a wide astronomy-literate community in a costeffective way.

The importance of effective and inclusive outreach efforts cannot be disputed (for example, see ref. ${ }^{2}$ ). Discriminating people based on their gender, age, race or ethnicity, can lead to their under-representation in the research community and diminish their contributions to science and astronomy ${ }^{3}$. For example, in the United States, between 2002 and 2012, close to 3\% of all astronomy doctoral students were members of minority communities ${ }^{4}$. Several efforts are underway to remedy this situation. Examples include projects directed to those with visual ${ }^{5}$ or hearing ${ }^{6}$ impairments. Another project uses the Japanese art form of manga to communicate astronomy to a more diverse audience?

In celebration of the IAU's 100th anniversary and its spirit of inclusivity, we organized the conference 100 Hours of Astronomy at the Border during 10-13 January 2019. The conference connected professional astronomers with amateur astronomers from all of the northern states of Mexico, with at least one professional astronomer representing every northern state, while making all of the talks accessible to everyone. Professional astronomers gave talks about galaxies, asteroids, exoplanets, astrobiology, black holes and star evolution. Astronomical observations were also carried out.

Our initiative aimed at kindling the appreciation for and interest in astronomy in Mexico, and consequently increasing the number of students in this scientific area. We employed technology to encourage the participation of people of all ages, genders and of underrepresented groups. Through this initiative, we attempted to solve the problem of exclusion due to distance and socioeconomic segregation.
In Mexico, a significant percentage of the population does not have an internet connection. By setting up a system of nodes and sub-nodes, we reached those in remote locations. Specifically, we created one node per state, which publicized our programme locally, had an open phone line to communicate onsite questions, monitored the quality of the broadcast and handled any technical difficulties (such as sound echo and slow internet connection due to weak Wi-Fi signals). The central node was located in Ciudad Juárez, Chihuahua, under the supervision of I.V.-P. and J.S., who used Skype to host a videoconference of all other local nodes. The Hermosillo, Sonora node was led by J.S.-M. and L.O. The rest of the nodes joined our network thanks to the hard work and enthusiasm of amateur astronomers: E.S. in Mulegé, Baja California, P.L.-P. in Monterrey, Nuevo León, and R.A. in Nuevo Laredo, Tamaulipas. The outreach goals and accessibility features of this project were conveyed in the talks presented by professional astronomers born or educated in northern Mexico. To maximize accessibility and dissemination, the talks were also broadcasted live on YouTube. Amateur astronomers in other cities were able to ask questions via Facebook and YouTube. Details about the talks and speakers are listed in the event programme.

Days before the event, we carried out a connection test with Skype between all speakers and nodes, but only four could join the videoconference at a time. As a result, during the actual conference, speakers were asked to contact the central node, through WhatsApp, thirty minutes before their talk, and then connect through Skype ten minutes before their presentation. For the webcast, the central node required three computers: one connected to a camera to share audio and video, one to monitor the social media comments, and one for the screen projection. Two microphones, an audio system and access ramps for people with disabilities in the auditoria were needed. For future events, we plan to have one person per node translating the talks to sign language or one in the central node on camera transmitting the translation.

Our joint effort was well received by audiences and the media alike. Our webcast had a higher impact on Friday (191 unique views) and on Saturday (148) rather than on Sunday (less than 100), according to YouTube statistics and our live audiences. For future events, consideration of the academic calendar might help increase the reach of these events. We estimate that our event reached a total of 270 people face to face and 351 online. Interestingly, we report that Nuevo Laredo had more children in the audience, followed by Ciudad Juárez and Monterrey, while Sonora and Baja California reported more teenagers.

During the event, Mulegé, Hermosillo, and Monterrey suffered from cloudy skies and therefore astronomical observations could not be carried out. Ciudad Juárez and Nuevo Laredo had clear skies. Future events should take into account the weather in northern Mexico, with January not being the optimal month for night-time observations.

Our pilot project is a first step to favour inclusion and access to information, and we will work to improve it. Our initiative encouraged professors in other academic fields to replicate the format. In addition, we are organizing similar activities, involving astronomers in Central America, the Caribbean and Mexico aimed to create a regional node of the IAU Office of Astronomy for Development. As a byproduct of the organization of this event, the Sonora team will collaborate with the Chihuahua node to organize a workshop on the analysis of asteroids.

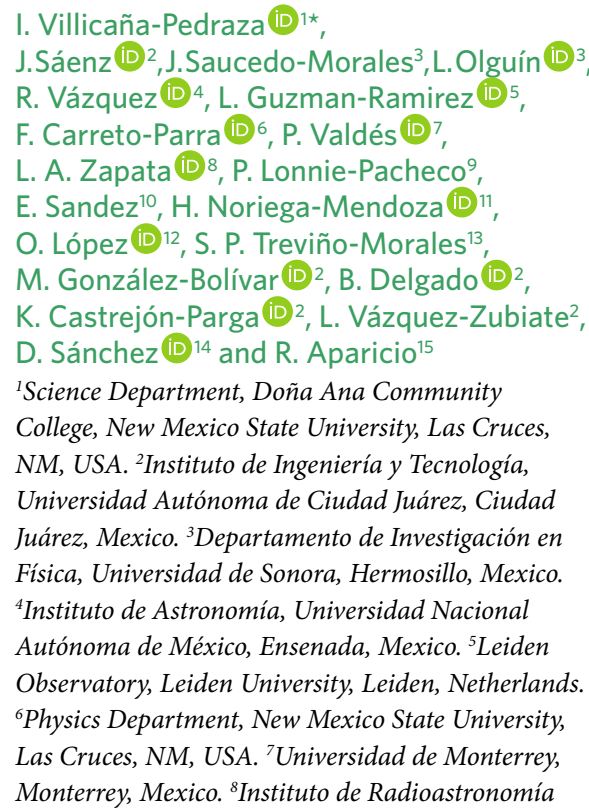


y Astrofísica, Universidad Nacional Autónoma de México, Morelia, Mexico. ${ }^{9}$ Planetario Alfa, Monterrey, Mexico. ${ }^{10}$ Instituto Tecnológico Superior de Mulegé, Santa Rosalía, Mexico. ${ }^{11}$ Physics Department, University of Texas at El Paso, El Paso, TX, USA. ${ }^{12}$ Instituto Nacional de Astrofísica, Óptica y Electrónica, Tonantzintla, Mexico. ${ }^{13}$ Department of Space, Earth and Environment, Chalmers University of Technology Onsala Space Observatory, Onsala, Sweden. ${ }^{14}$ Facultad de Ciencias Naturales, Universidad Autónoma de Querétaro, Santiago de Querétaro, Mexico. ${ }^{15}$ Cosmo Glaretum, Nuevo Laredo, Mexico.

*e-mail:ilhui7@nmsu.edu

Published online: 26 August 2019

https://doi.org/10.1038/s41550-019-0875-z

References

1. Nat. Astron. 3, 111 (2019)

2. Pérez-Montero, E. Nat. Astron. 3, 114-115 (2019).

3. Knezek, P. Nat. Astron. 1, 0151 (2017).

4. Zastrow, M. Astronomy is losing women three times faster than men. Nature (18 October 2018).
5. Gruber, D. CAPjournal 25, 18 (2019).

6. De Leo-Winkler, M. A. et al. J. Sci Educ. Technol. 28 222-230 (2019).

7. Dall'Olio, D. \& Ranalli, P. CAPjournal 24, 7 (2018).

Acknowledgements

The authors thank A. Ortiz-Gil for her guidance, and the IT staff of the Universidad Autónoma de Ciudad Juárez. S.P.T.-M. acknowledges the European Union's Horizon 2020 research and innovation programme for funding support given under grant agreement no. 639459 (PROMISE). I.V.-P. acknowledges L. Binette, Y. Rito, R. Pankayatselvan, J. Butler, R. Walterbos, R. Armendariz and A. Bruce for their support. 